\title{
Additive Schemes for Systems of Time-Dependent Equations of Mathematical Physics
}

\author{
Alexander Samarskii and Petr Vabishchevich \\ Institute for Mathematical Modeling, RAS \\ 4-A Miusskaya Square, 125047 Moscow, Russia \\ vab@imamod.ru
}

\begin{abstract}
Additive difference schemes are derived via a representation of an operator of a time-dependent problem as a sum of operators with a more simple structure. In doing so, transition to a new time level is performed as a solution of a sequence of more simple problems. Such schemes in various variants are employed for approximate solving complicated time-dependent problems for PDEs. In the present work construction of additive schemes is carried out for systems of parabolic and hyperbolic equations of second order. As examples there are considered dynamic problems of the elasticity theory for materials with variable properties, dynamics problems for an incompressible fluid with a variable viscosity, general 3D problems of magnetic field diffusion.
\end{abstract}

\section{Introduction}

Nowadays, various classes of additive difference schemes are developed by splitting the problem operator into certain components [1 2 3 4 45]. Classical examples of additive difference schemes are so called economical difference schemes for multi-dimensional problems of mathematical physics. The simplest examples of these economical schemes are the well-known alternating direction schemes, locally-one-dimensional schemes etc. In this case we have additive difference schemes where the splitting is done with respect to the separate directions.

Very often the components of the splitting operator are connected with description of differed components of the process under investigation. For instance, in continuum mechanics the process of transport of a substance can be decomposed into transport due to medium motion and transport by means of diffusion. To highlight such a peculiarity of the problem, this decomposition is referred to as splitting with respect to physical processes.

Domain decomposition methods are in common use in constructing computational algorithms for the solution of time-dependent problems for PDEs on modern parallel computers [5,6]. In this case, a particular processor solves boundary value problem in a separate subdomain. The corresponding additive difference schemes are referred to as regional-additive ones.

Numerical solution of systems of time-dependent PDEs is often required in applications of mathematics to scientific, industrial and environmental problems. 
As a rule, the unknown variables are included into several equations, what makes impossible to find one component independently from others. In this case, the operator corresponding to the system can be split into more simple operators, connected with separate components of the solution. Thus, the transition to a new time level can be based on solving consecutively separate problems for each of the component of the solution. Possibilities for constructing such additive schemes are considered in the present work. Hydrodynamics problems for an incompressible fluid with a variable viscosity and for diffusion of a magnetic field are considered as typical examples.

\section{Problem Formulation}

Let us consider real grid functions $y$ from a finite-dimensional real Hilbert space $H$. In this space we have the dot product and norm $(\cdot, \cdot), \quad\|y\|=\sqrt{(y, y)}$, respectively. For an operator $D=D^{*}>0$ we introduce $H_{D}$ as space $H$ with dot product $(y, w)_{D}=(D y, w)$ and norm $\|y\|_{D}=\sqrt{(D y, y)}$.

We will search solution $u(t) \in H$ of the Cauchy problem for the following evolutionary equation of first order

$$
\begin{aligned}
\frac{d u}{d t}+A u & =f(t), \quad 0<t \leq T, \\
u(0) & =u_{0} .
\end{aligned}
$$

Let us consider the simplest case of positive self-adjoint steady-state operator $A$, i.e. $A \neq A(t)=A^{*}>0$.

In a similar way, the Cauchy problem for the evolutionary equation of second order is formulated. In this case we search $u(t) \in H$ from the equation

$$
\begin{aligned}
\frac{d^{2} u}{d t^{2}}+A u & =f(t), \quad 0<t \leq T, \\
u(0) & =u_{0}, \\
\frac{d u}{d t}(0) & =u_{1} .
\end{aligned}
$$

The following estimate, concerning stability of the solution with respect to the initial data and to the RHS, is fulfilled for the above problem (11),(2) (it will further serve us as a checkpoint in constructing difference schemes).

Lemma 1. The following estimate holds for problem (1), (2)

$$
\|u(t)\| \leq\left\|u_{0}\right\|+\int_{0}^{t}\|f(s)\| d s .
$$

In a similar way, it is possible to formulate a statement concerning stability of the solution of problem(3) - (5) with respect to the initial data and to the right hand side. 
Lemma 2. The following estimate holds for problem (3) - (5)

$$
\|u(t)\|_{*} \leq\left\|u_{0}\right\|_{A}+\left\|u_{1}\right\|+\int_{0}^{t}\|f(s)\| d s,
$$

where

$$
\|u(t)\|_{*}^{2} \equiv\|u\|_{A}^{2}+\left\|\frac{d u}{d t}\right\|^{2} .
$$

Our aim is to construct additive schemes for problems (1), (22) and (3) - (5). Assume that the operator $A$ has an additive representation

$$
A=\sum_{\alpha=1}^{p} A^{(\alpha)}, \quad A^{(\alpha)} \neq A^{(\alpha)}(t)=\left(A^{(\alpha)}\right)^{*} \geq 0, \quad \alpha=1,2, \ldots, p .
$$

Additive difference schemes are derived on the basis of representation (6), where a transition from one time-level $t^{n}$, to the next one $t^{n+1}=t^{n}+\tau$ (here $\tau>0$ time-increment), is connected with solving problems for the separate operators $A^{(\alpha)}, \alpha=1,2, \ldots, p$. Thus, the initial problem is decomposed into $p$ more simple subproblems.

\section{Some Classes of Additive Schemes}

\subsection{Schemes of Component-wise Splitting}

Additive difference schemes for problems with splitting into three and more operators (which are pair-wise non-commutative ones), are constructed on the basis of the concept of the additive approximation. Schemes with componentwise splitting (locally one-dimensional schemes) serve as a prototype in this case. The following difference schemes are employed for problem (1), (2), (6)

$$
\frac{y^{n+\alpha / p}-y^{n+(\alpha-1) / p}}{\tau}+A^{(\alpha)}\left(\sigma_{\alpha} y^{n+\alpha / p}+\left(1-\sigma_{\alpha}\right) y^{n+(\alpha-1) / p}\right)=f_{\alpha}^{n},
$$

$\alpha=1,2, \ldots, p, \quad n=0,1, \ldots$, where $f^{n}=\sum_{\alpha=1}^{p} f_{\alpha}^{n}$.

For $\sigma_{\alpha} \geq 0.5$, the component-wise splitting scheme (7) is unconditionally stable. Let us derive the corresponding a priori estimate for stability with respect to the initial data and to the right hand side. A special representation is used for the right hand sides $f_{\alpha}^{n}, \alpha=1,2, \ldots, p$ :

$$
f_{\alpha}^{n}=\stackrel{\circ}{f_{\alpha}^{n}}+\stackrel{*}{f_{\alpha}^{n}}, \quad \alpha=1,2, \ldots, p, \quad \sum_{\alpha=1}^{p} \stackrel{\circ}{f_{\alpha}^{n}}=0 .
$$

Such a form of the right hand side is essential in the consideration of the problem for the error of the additive scheme. The following statement holds for the component-wise splitting scheme. 
Theorem 1. For $0.5 \leq \sigma_{\alpha} \leq 2, \alpha=1,2, \ldots, p$ and $\tau>0$, the following a priori estimate holds for the solution of the Cauchy problem for difference equations (6), (8)

$$
\left\|y^{n+1}\right\| \leq\left\|u_{0}\right\|+\sum_{k=0}^{n} \tau \sum_{\alpha=1}^{p}\left(\left\|f_{\alpha}^{*}\right\|+\tau\left\|A^{(\alpha)} \sum_{\beta=\alpha}^{p} \stackrel{\circ}{f_{\beta}^{k}}\right\|\right) .
$$

\subsection{Additively-Averaged Schemes}

Additively-averaged schemes, based on component-wise splitting can be used successfully on modern parallel computers. In this case the transition to a new time level is performed as follows:

$$
\frac{y_{\alpha}^{n+1}-y^{n}}{p \tau}+A^{(\alpha)}\left(\sigma_{\alpha} y_{\alpha}^{n+1}+\left(1-\sigma_{\alpha}\right) y^{n}\right)=f_{\alpha}^{n},
$$

$\alpha=1,2, \ldots, p, \quad n=0,1, \ldots, y^{n+1}=\frac{1}{p} \sum_{\alpha=1}^{p} y_{\alpha}^{n+1}$.

The stability conditions for these schemes are the same as for standard scheme of component-wise splitting. Similarly to Theorem 1, the following statement can be proved.

Theorem 2. For $\sigma_{\alpha} \geq 0.5, \alpha=1,2, \ldots, p$ and any $\tau>0$, the following $a$ priori estimate holds for the solution of (6), (2)

$$
\left\|y^{n+1}\right\| \leq\left\|u_{0}\right\|+\sum_{k=0}^{n} \tau \sum_{\alpha=1}^{p}\left(\left\|f_{\alpha}^{*}\right\|+p \tau \sigma_{\alpha}\left\|A^{(\alpha)} \stackrel{\circ}{f_{\alpha}^{k}}\right\|\right) .
$$

A potential advantage of the additively-averaged scheme (9) is connected with the fact that it is possible to perform parallel evaluation of grid functions $y_{\alpha}^{n+1}$, $\alpha=1,2, \ldots, p$.

\subsection{Regularized Additive Schemes}

It is convenient to construct additive schemes on the basis of the regularization principle for the difference schemes. An example is the additive scheme constructed by perturbing each separate operator in the additive representation (6):

$$
\frac{y^{n+1}-y^{n}}{\tau}+\sum_{\alpha=1}^{p}\left(E+\sigma_{\alpha} \tau A^{(\alpha)}\right)^{-1} A^{(\alpha)} y^{n}=f^{n}, \quad n=0,1, \ldots
$$

Theorem 3. For $\sigma_{\alpha} \geq p / 2, \alpha=1,2, \ldots, p$ and any $\tau>0$, the following a priori estimate holds for the solution of equation (6), (10)

$$
\left\|y^{n+1}\right\| \leq\left\|u_{0}\right\|+\sum_{k=0}^{n} \tau\left\|f^{k}\right\|
$$


The considered regularized scheme (10) is closely connected with the above considered additively-averaged scheme. To illustrate this fact, let us introduce fictitious grid unknowns $y_{\alpha}^{n+1}, \alpha=1,2, \ldots, p$. These functions have no independent sense and are used for auxiliary purposes. Let us rewrite the scheme (10) as follows:

$$
\frac{y_{\alpha}^{n+1}-y^{n}}{p \tau}+\left(E+\sigma \tau A^{(\alpha)}\right)^{-1} A^{(\alpha)} y^{n}=f_{\alpha}^{n},
$$

$\alpha=1,2, \ldots, p, \quad n=0,1, \ldots, y^{n+1}=\frac{1}{p} \sum_{\alpha=1}^{p} y_{\alpha}^{n+1}$.

Thus, we again obtain an additively-averaged scheme, but in this case it is constructed without using the concept of the additive approximation. This scheme differs from the early presented scheme (9) in approximation of the right hand sides.

\subsection{Second-Order Equation}

Certain problems arise in constructing operator-splitting schemes for evolutionary equations of second order. Let us discuss now some classes of regularized additive schemes (so called full approximation schemes) for evolutionary equations of second order. The multiplicative regularization for the problem (3) - (5) results in the scheme

$$
\frac{y^{n+1}-2 y^{n}+y^{n-1}}{\tau^{2}}+\sum_{\alpha=1}^{p}\left(E+\sigma \tau^{2} A^{(\alpha)}\right)^{-1} A^{(\alpha)} y^{n}=f^{n}, \quad n=1,2, \ldots
$$

Theorem 4. The additive difference scheme (11) for the problem (3) - (5) is unconditionally stable for $\sigma_{\alpha} \geq p / 4, \alpha=1,2, \ldots, p$.

The scheme (11) can be implemented as follows:

$$
\left(E+\sigma \tau^{2} A^{(\alpha)}\right) \frac{y_{\alpha}^{n+1}-2 y^{n}+y^{n-1}}{p \tau^{2}}+A^{(\alpha)} y^{n}=\frac{1}{p}\left(E+\sigma \tau^{2} A^{(\alpha)}\right) f^{n},
$$

$\alpha=1,2, \ldots, p, \quad n=1,2, \ldots, y^{n+1}=\frac{1}{p} \sum_{\alpha=1}^{p} y_{\alpha}^{n+1}$.

Thus we obtain a special additively-averaged scheme.

\section{Alternating Triangle Method}

The alternating triangle method has been developed in 7] for solving the Cauchy problem for linear ODEs with a symmetric matrix. It is based on splitting of the equation matrix into two triangular matrices. A general description of the alternating triangle method is presented below along with the discussion of possibilities for constructing new classes of additive alternating triangle schemes. Namely, this class of additive schemes is convenient for solving boundary value problems for systems of time-dependent equations of mathematical physics. 


\subsection{General Description of the Alternating Triangle Method}

Let us consider Cauchy problem (1), (2) with a time-independent self-adjoint positive definite operator $A$. The alternating triangle method is defined by the two-component additive splitting

$$
A=A^{(1)}+A^{(2)}>0, \quad\left(A^{(1)}\right)^{*}=A^{(2)} .
$$

Let (1), (2) be the operator formulation of the Cauchy problem for the system of linear ODEs of first order

$$
\begin{gathered}
\frac{d u_{i}(t)}{d t}+\sum_{j=1}^{m} a_{i j} u_{j}(t)=f_{i}(t), \quad t>0, \\
u_{i}(0)=u_{i}^{0}, \quad i=1,2, \ldots, m .
\end{gathered}
$$

Here $u=\left\{u_{1}, u_{2}, \ldots u_{m}\right\}$ stands for the vector of unknowns, $f=\left\{f_{1}, f_{2}, \ldots f_{m}\right\}$ - the specified vector of right hand sides, and $A=\left\{a_{i j}\right\}$ - the symmetric real matrix with elements $a_{i j}=a_{j i}, i, j=1,2, \ldots, m$.

Under the above conditions, the matrix $A$ of the problem (13), (14) is considered as a self-adjoint linear operator in a finite-dimensional Hilbert (Euclidean) space $H=l_{2}$ with dot product $(y, v)=\sum_{i=1}^{m} y_{i} v_{i}$ and norm $\|y\|=\sqrt{(y, y)}$.

For the elements of the matrices

$$
A^{(\alpha)}=\left\{a_{i j}^{(\alpha)}\right\}, \quad \alpha=1,2,
$$

in correspondence with the decomposition (12), we have

$$
a_{i j}^{(1)}=\left\{\begin{array}{l}
a_{i j}, \quad i<j, \\
\frac{1}{2} a_{i i}, i=j, \\
0, \quad i>j,
\end{array} \quad a_{i j}^{(2)}= \begin{cases}0, & i<j, \\
\frac{1}{2} a_{i i}, & i=j, \\
a_{i j}, & i>j .\end{cases}\right.
$$

Thus, the matrix $A$ is decomposed into two triangular matrices.

The standard variant of the alternating triangle method employs the alternating direction scheme by Peaceman-Rachford [8] for solving the problem (11), (2), (12)

$$
\begin{gathered}
\frac{y^{n+1 / 2}-y^{n}}{0.5 \tau}+A^{(1)} y^{n+1 / 2}+A^{(2)} y^{n}=f^{n}, \\
\frac{y^{n+1}-y^{n+1 / 2}}{0.5 \tau}+A^{(1)} y^{n+1 / 2}+A^{(2)} y^{n+1}=f^{n} .
\end{gathered}
$$

Implementation of the above additive schemes is connected with the consecutive inversion of the upper and the lower triangular matrices, what explains the name "the alternating triangle method" for these additive schemes. 


\subsection{Equations of First Order}

Additive schemes of the alternating triangle method can be investigated in the most complete way using results of the general theory of stability for operatordifference schemes 29]. Let us rewrite the two-level factorized scheme of the alternating triangle method in the canonical form

$$
\begin{gathered}
B \frac{y^{n+1}-y^{n}}{\tau}+A y^{n}=f^{n}, \quad t_{n} \in \omega_{\tau} \\
B=\left(E+\sigma \tau A^{(1)}\right)\left(E+\sigma \tau A^{(2)}\right) .
\end{gathered}
$$

The scheme (17), (18) is equivalent to the scheme (15), (16) when the weight parameter $\sigma$ equals one half: $\sigma=0.5$.

Theorem 5. Factorized scheme of alternating triangle method (12), (17), (18) is unconditionally stable for $\sigma \geq 0.5$ in $H_{A}$. The following a priori estimate holds

$$
\left\|y^{n+1}\right\|_{A}^{2} \leq\left\|y^{0}\right\|_{A}^{2}+\frac{1}{2} \sum_{k=0}^{n} \tau\left\|f^{k}\right\|^{2} .
$$

The alternating triangle additive scheme for three-level operator-difference schemes is constructed in a similar way. Schemes from this class are also constructed for problems with non-selfadjoint operators in the case of subordination of the skew-symmetric part of the operator [5].

\subsection{Equation of Second Order}

In constructing additive schemes for the Cauchy problem for evolutionary equation of second order (3) - (5), the emphasize is on the additive alternating triangle schemes.

For three-level schemes

$$
D \frac{y_{n+1}-2 y_{n}+y_{n-1}}{\tau^{2}}+A y_{n}=\varphi_{n}, \quad n=1,2, \ldots
$$

with splitting (12), we define a factorized operator

$$
D=\left(E+\sigma \tau^{2} A^{(1)}\right)\left(E+\sigma \tau^{2} A^{(2)}\right) .
$$

Theorem 6. Factorized scheme of alternating triangle method (12), (19), (20) is unconditionally stable for $\sigma \geq 0.25$.

This statement follows from the estimate

$$
D=D^{*} \geq E+\sigma \tau^{2} A
$$

and from the general results of the stability theory for three-level operatordifference schemes [2]. 


\section{$5 \quad$ Hydrodynamics Problems for an Incompressible Fluid with Variable Viscosity}

In modeling flows of an inhomogeneous fluid, in particular, in solving hydrodynamics problems with free boundaries, it is necessary to consider problems with a variable viscosity. The primary peculiarities of such problems are connected with the fact, that equations for the particular components of the velocity are strongly coupled (through the principal derivatives). There is no such a coupling in the problems with a constant viscosity. Let us recall, that in the constant viscosity case, the (linearized) system of momentum equations can be naturally decomposed and it is possible to solve independently equations for the velocity components. The emphasis in the case of variable viscosity is on constructing special additive schemes. They are based on splitting with respect to the physical processes and take into account the peculiarities of the problems with variable viscosity [10].

\subsection{Problem Formulation}

Let $\varrho$ be the density, $p$ - the pressure, $\mathbf{u}$ - the velocity and $\mu$ - the viscosity of an incompressible fluid. The momentum equation has the form

$$
\varrho\left(\frac{\partial \mathbf{u}}{\partial t}+(\mathbf{u} \cdot \operatorname{grad}) \mathbf{u}\right)+\operatorname{grad} p=\operatorname{Div} \sigma+\varrho \mathbf{f},
$$

where $\sigma$ stands for the viscous stresses tensor and $\mathbf{f}$ stands for the volumetric force (e.g., the buoyant force in free convection problems).

In the case of a Newtonian fluid, the viscous stresses tensor has the following coordinate-wise representation:

$$
\sigma_{i j}=\mu\left(\frac{\partial u_{i}}{\partial x_{j}}+\frac{\partial u_{j}}{\partial x_{i}}\right)
$$

In addition, for an incompressible fluid the continuity equation has the form

$$
\operatorname{div} \mathbf{u}=0
$$

For simplicity, let us consider motion of a fluid with a constant density. Thus, instead of (21) we employ the equation

$$
\frac{\partial \mathbf{u}}{\partial t}+(\mathbf{u} \cdot \operatorname{grad}) \mathbf{u}+\operatorname{grad} p^{\prime}=\operatorname{Div} \sigma^{\prime}+\varrho \mathbf{f}^{\prime},
$$

where the pressure, the tensor of viscous stresses and the force are normalized by the density in such a way that, for instance,

$$
\sigma_{i j}=\nu\left(\frac{\partial u_{i}}{\partial x_{j}}+\frac{\partial u_{j}}{\partial x_{i}}\right)
$$

where $\nu=\mu / \varrho$ is the kinematic viscosity. 


\subsection{The 2D Problem}

The detailed consideration will be conducted for planar-parallel flows. Let $\mathbf{x}=$ $\left(x_{1}, x_{2}\right), \mathbf{v}=\left(v_{1}, v_{2}\right)$, then equations (22), (23) in the coordinate-wise representation take the following form

$$
\begin{gathered}
\frac{\partial v_{1}}{\partial t}+v_{1} \frac{\partial v_{1}}{\partial x_{1}}+v_{2} \frac{\partial v_{1}}{\partial x_{2}}+\frac{\partial p^{\prime}}{\partial x_{1}}= \\
=\frac{\partial}{\partial x_{1}}\left(2 \nu \frac{\partial v_{1}}{\partial x_{1}}\right)+\frac{\partial}{\partial x_{2}}\left(\nu \frac{\partial v_{1}}{\partial x_{2}}\right)+\frac{\partial}{\partial x_{2}}\left(\nu \frac{\partial v_{2}}{\partial x_{1}}\right)+f_{1}^{\prime}(\mathbf{x}, t), \\
\frac{\partial v_{2}}{\partial t}+v_{1} \frac{\partial v_{2}}{\partial x_{1}}+v_{2} \frac{\partial v_{2}}{\partial x_{2}}+\frac{\partial p^{\prime}}{\partial x_{2}}= \\
=\frac{\partial}{\partial x_{1}}\left(\nu \frac{\partial v_{1}}{\partial x_{2}}\right)+\frac{\partial}{\partial x_{1}}\left(\nu \frac{\partial v_{2}}{\partial x_{1}}\right)+\frac{\partial}{\partial x_{2}}\left(2 \nu \frac{\partial v_{2}}{\partial x_{2}}\right)+f_{2}^{\prime}(\mathbf{x}, t), \\
\frac{\partial v_{1}}{\partial x_{1}}+\frac{\partial v_{2}}{\partial x_{2}}=0 .
\end{gathered}
$$

Using the representation (24) - (26), it is more easy to see the coupling between components $v_{\alpha}, \alpha=1,2$ in the right hand side of the momentum equation.

\subsection{Operator of Viscous Stresses}

Let us define the standard Hilbert space $\mathcal{H}=L_{2}(\Omega)$ with the dot product and norm

$$
(u, v)=\int_{\Omega} u(\mathbf{x}) v(\mathbf{x}) d \mathbf{x}, \quad\|u\|=(u, u)^{1 / 2} .
$$

For the $2 \mathrm{D}$ vectors $\mathbf{u}, \mathbf{v}$ we introduce the Hilbert space $\mathcal{H}_{2}=\mathcal{H} \oplus \mathcal{H}$ with dot product given by

$$
(\mathbf{u}, \mathbf{v})=\left(u_{1}, v_{1}\right)+\left(u_{2}, v_{2}\right)
$$

On the set of vector functions $\mathbf{v}$ equal to zero on $\partial \Omega$, we define the operator $\mathcal{N}$,

$$
\mathcal{N} \mathbf{v}=-\operatorname{Div} \sigma
$$

In accordance with (24), (25), the operator $\mathcal{N}$ is nothing but the matrix

$$
\mathcal{N}=\left(\begin{array}{ll}
\mathcal{N}_{11} & \mathcal{N}_{12} \\
\mathcal{N}_{21} & \mathcal{N}_{22}
\end{array}\right)
$$


where

$$
\begin{aligned}
& \mathcal{N}_{11} v_{1}=-\frac{\partial}{\partial x_{1}}\left(2 \nu \frac{\partial v_{1}}{\partial x_{1}}\right)+\frac{\partial}{\partial x_{2}}\left(\nu \frac{\partial v_{1}}{\partial x_{2}}\right), \\
& \mathcal{N}_{12} v_{2}=-\frac{\partial}{\partial x_{2}}\left(\nu \frac{\partial v_{2}}{\partial x_{1}}\right), \\
& \mathcal{N}_{21} v_{1}=-\frac{\partial}{\partial x_{1}}\left(\nu \frac{\partial v_{1}}{\partial x_{2}}\right), \\
& \mathcal{N}_{22} v_{2}=-\frac{\partial}{\partial x_{1}}\left(\nu \frac{\partial v_{2}}{\partial x_{1}}\right)+\frac{\partial}{\partial x_{2}}\left(2 \nu \frac{\partial v_{2}}{\partial x_{2}}\right) .
\end{aligned}
$$

It is easy to see that

$$
(\mathcal{N} \mathbf{v}, \mathbf{u})=(\mathbf{v}, \mathcal{N} \mathbf{u})
$$

i.e. operator $\mathcal{N}=\mathcal{N}^{*}>0$ in $\mathcal{H}_{2}$.

In constructing unconditionally stable difference schemes, it is necessary to derive schemes where terms with viscous stresses are implicitly discretized. On the other hand, the fully implicit treatment of this operator is not convenient due to the above mentioned coupling of the velocity components. So, it is necessary to select an operator that is close to $\mathcal{N}$ but is more convenient in computational implementation. It is natural to take the following operator

$$
\mathcal{D}=\left(\begin{array}{cc}
\mathcal{D}_{11} & 0 \\
0 & \mathcal{D}_{22}
\end{array}\right),
$$

where

$$
\begin{aligned}
& \mathcal{D}_{11} v_{1}=-\frac{\partial}{\partial x_{1}}\left(\nu \frac{\partial v_{1}}{\partial x_{1}}\right)+\frac{\partial}{\partial x_{2}}\left(\nu \frac{\partial v_{1}}{\partial x_{2}}\right) \\
& \mathcal{D}_{22} v_{2}=-\frac{\partial}{\partial x_{1}}\left(\nu \frac{\partial v_{2}}{\partial x_{1}}\right)+\frac{\partial}{\partial x_{2}}\left(\nu \frac{\partial v_{2}}{\partial x_{2}}\right) .
\end{aligned}
$$

Thus

$$
\mathcal{D} \mathbf{v}=-\operatorname{div}(\nu \operatorname{grad} \mathbf{v})
$$

It is easy to show that operators $\mathcal{N}$ and $\mathcal{D}$ are energy equivalent. Namely, the following estimate holds

$$
\nu(\mathcal{D} \mathbf{v}, \mathbf{v}) \leq(\mathcal{N} \mathbf{v}, \mathbf{v}) \leq 2 \nu(\mathcal{D} \mathbf{v}, \mathbf{v})
$$

The facts that operator $\mathcal{N}$ is self-adjoint and positive definite in space $\mathcal{H}_{2}$ and, moreover, it is energy equivalent to the Laplace operator demonstrate its most important properties. Just these peculiarities will be preserved in constructing of the discrete analogs of the differential operators.

For of the numerical implementation we will employ representation of the operator matrix $\mathcal{N}$ as a sum of two operators:

$$
\mathcal{N}=\mathcal{N}^{(1)}+\mathcal{N}^{(2)}, \quad\left(\mathcal{N}^{(1)}\right)^{*}=\mathcal{N}^{(2)} .
$$


Taking into account the expression for the operator $\mathcal{N}$, we have

$$
\mathcal{N}^{(1)}=\left(\begin{array}{cc}
\frac{1}{2} \mathcal{N}_{11} & 0 \\
\mathcal{N}_{21} & \frac{1}{2} \mathcal{N}_{22}
\end{array}\right), \quad \mathcal{N}^{(2)}=\left(\begin{array}{cc}
\frac{1}{2} \mathcal{N}_{11} & \mathcal{N}_{12} \\
0 & \frac{1}{2} \mathcal{N}_{22}
\end{array}\right) .
$$

In using additive schemes calculations are connected with inversion of operators $E+\sigma \tau \mathcal{N}^{(\alpha)}, \alpha=1,2$ (without convective transport). Thus, we have the decomposed system of equations with separate solution of elliptic boundary value problems for particular components of the velocity.

In fact, we have two closely related possibilities. The first possibility is connected with constructing difference schemes on the basis of regularizer $\mathcal{D}$, the second one - on using triangular splitting (27). Viscous terms are split in both cases.

\subsection{Additive Difference Schemes}

Differential equations (22), (23) can be written as a single equation

$$
\frac{d \mathbf{v}}{d t}+\mathcal{V} \mathbf{v}+\mathcal{P} \mathbf{v}+\mathcal{N} \mathbf{v}=\mathbf{f}^{\prime}
$$

for solenoidal functions $\mathbf{v} \in \mathcal{H}_{2}$ (functions that satisfy condition (22)). Here $\mathcal{V}$ is the convective transport operator, whereas $\mathcal{P}$ is the operator connected with the pressure. The boundary and the initial conditions are treated in the standard way.

Let us construct the simplest difference schemes for equation (27), based on the uniform integration in time with increment $\tau>0$. As the basis we consider the scheme where the convective transport is taken from the previous timelevel. Let us derive regularized additive schemes. Unfortunately, the traditional schemes of ADI-type are not possible here.

Using the regularizer $\mathcal{D}$, we can employ the scheme

$$
\begin{aligned}
\frac{\mathbf{v}^{n+1}-\mathbf{v}^{n}}{\tau}+\mathcal{V}^{n} \mathbf{v}^{n}+\left(E+\sigma_{1} \tau \mathcal{D}\right)^{-1} \mathcal{N}\left(E+\sigma_{1} \tau \mathcal{D}\right)^{-1} \mathbf{v}^{n} & + \\
& +\left(E+\sigma_{2} \tau \mathcal{P}\right)^{-1} \mathcal{P} \mathbf{v}^{n}=\mathbf{f}_{n}^{\prime} .
\end{aligned}
$$

The proposed scheme can be implemented in various ways. For example, it is possible to introduce some auxiliary function $\mathbf{v}^{n+1 / 2}$, which is to be determined from the equation

$$
\frac{\mathbf{v}^{n+1 / 2}-\mathbf{v}^{n}}{\tau}+\mathcal{V}^{n} \mathbf{v}^{n}+\left(E+\sigma_{1} \tau \mathcal{D}\right)^{-1} \mathcal{N}\left(E+\sigma_{1} \tau \mathcal{D}\right)^{-1} \mathbf{v}^{n}=\mathbf{f}_{n}^{\prime}
$$

In such a way we calculate the transport due to the convection, due to viscosity and due to volumetric forces.

The second stage is the calculation of the transport due to the pressure:

$$
\frac{\mathbf{v}^{n+1}-\mathbf{v}^{n+1 / 2}}{\tau}+\left(E+\sigma_{2} \tau \mathcal{P}\right)^{-1} \mathcal{P} \mathbf{v}^{n}=0
$$


In fact, schemes of such a type are in common use. The primary peculiarity is in calculation of the first stage. This complicated construction is necessary for deriving a stable scheme. Implementation consists in double solution of elliptic problem $E+\sigma_{1} \tau \mathcal{D}$. Such an increasing of the computational costs is not essential, compare to the advantages of the scheme.

In a similar way we can design schemes with triangular decomposition (27). For instance, an analog of scheme (28) will be the following one

$$
\begin{aligned}
\frac{\mathbf{v}_{n+1}-\mathbf{v}_{n}}{\tau}+\left(E+\sigma_{1} \tau \mathcal{N}^{(1)}\right)^{-1}\left(\mathcal{N}+\mathcal{V}_{n}\right) & \left(E+\sigma_{1} \tau \mathcal{N}^{(2)}\right)^{-1} \mathbf{v}_{n}+ \\
& +\left(E+\sigma_{2} \tau \mathcal{P}\right)^{-1} \mathcal{P} \mathbf{v}_{n}=\mathbf{f}_{n}^{\prime}
\end{aligned}
$$

The advantage of scheme (29) compare to scheme (28) consists in the fact, that only one elliptic problem for evaluation of the velocity components is solved here at each time-level (but this elliptic problem is slightly more complicated).

\section{Generalizations}

In a similar way we can construct additive operator-difference schemes for solving the system of equation for the diffusion of a magnetic field. Induction of a magnetic field is governed by the equation

$$
\frac{\partial \mathbf{B}}{\partial t}-\operatorname{rot}\left(\frac{1}{\sigma} \operatorname{rot} \mathbf{B}\right)=0 .
$$

In the general $3 \mathrm{D}$ case it is no possible to formulate boundary value problems for particular components of vector B. For equation (30) we can design additive alternating triangular difference schemes.

Evolutionary equations of second order arise in considering dynamic problems of the elasticity theory. Some possibilities of designing additive operatordifference schemes for this case are discussed in the work 11.

\section{Acknowledgments}

This research was supported by Russian Foundation for Basic Research under grant No. 02-01-00555.

\section{References}

1. Yanenko, N. N.: The Method of Fractional Steps. Springer-Verlag, New York (1967)

2. Samarskii, A. A.: The Theory of Difference Schemes. Marcell Dekker (2001)

3. Marchuk, G. I.: Splitting and alternating direction methods. In: Ciarlet,P. G., Lions, J.-L. (eds): Handbook of Numerical Analysis, Vol. 1. North-Holland, Amsterdam (1990) 197-462 
4. Samarskii, A. A., Vabishchevich, P. N.: Computational Heat Transfer, Vol.1,2. Wiley, Chichester (1995)

5. Samarskii, A. A., Vabishchevich, P. N.: Additive Schemes for Problems of Mathematical Physics. Nauka, Moscow (1999) (in Russian)

6. Samarskii, A. A., Vabishchevich, P. N., Matus, P. P.: Difference Schemes with Operator Factors. Minsk (1998) (in Russian)

7. Samarskii, A. A.: An economical algorithm for numerical solution of systems of differential and algebraic equations. Zhurnal Vychislitel'noi Matematiki i Matematicheskoi Fiziki. 4 (1964) 580-585 (in Russian)

8. Peaceman, D. W.,Rachford, H. H.: The numerical solution of parabolic and elliptic differential equations. J. SIAM. 3 (1955) 28-41

9. Samarskii, A. A., Gulin, A. V.: Stability of Difference Schemes. Nauka, Moscow (1973) (in Russian)

10. Vabishchevich, P. N., Samarskii, A. A.: Solution of problems of incompressible fluid dynamics with variable viscosity. Zhurnal Vychislitel'noi Matematiki i Matematicheskoi Fiziki. 40 (2000) 1813-1822 (in Russian)

11. Lisbona, F. L., Vabishchevich, P. N.: Operator-splitting schemes for solving elasticity problems. Comput. Methods in Applied Math. 1 (2001) 188-198 